\title{
Orbitopathy as a manifestation of Immunoglobulin-G4-related disease \\ - case report
}

Agnieszka Zwolak ${ }^{1,2}$, Marta Dudzińska ${ }^{1}$, Joanna Świrska ${ }^{1,2}$, Joanna Malicka ${ }^{2}$, Jadwiga Daniluk ${ }^{1}$, Jerzy S. Tarach ${ }^{2}$

${ }^{1}$ Chair of Internal Medicine and Department of Internal Medicine in Nursing, Medical University in Lublin, Poland

${ }^{2}$ Department of Endocrinology, Medical University in Lublin, Poland

Introduction:Immunoglobulin-G4-related disease (IgG4RD) is a multidisciplinary problem due to plasmatic cells infiltration and areas of fibrosis in the affected tissues. In IgG4RD, elevation of serum IgG4 immunoglobulins is present. Clinically, the disease is mainly manifested as: autoimmune pancreatitis, salivary gland involvement, sclerosing cholangitis, lymphadenopathy, lachrymal gland enlargement, retroperitoneal fibrosis or orbital pseudotumor. The simultaneous involvement of multiple organs is common. Treatment is based on glucocorticosteroids. In case of recurrence or resistance to steroids, immunosuppresive therapy, involving disease-modifying antirheumatic drugs or rituximab is used.

Material and methods: The aim of the study was to present a patient with orbitopathy in whom in the course of diagnostic procedure, IgG4related eye disease was detected.

Case report: A 68-year old man, without past history of any chronic diseases, was admitted to the outpatient endocrinology clinic because of increased protrusion of both eyeballs with swallowing conjunctivitis, eyelids redness and retro orbital pain, without impairment of vision. The MRI scans revealed enlargement and swallowing of all periorbital muscles (Fig.1).

The thyroid function was normal. Futermore, as the antythyroid immunoglobulins were not present, the Graves orbitopathy was ruled out. (Tab.1). No significant pathology in the ultrasound of thyroid gland was found either.

Since elevated serum total $\lg G$ and $\operatorname{lgG} 4$ was stated, the lgG4related disease was recognized and therefore systemic glicocorticosteroid therapy (methylprednisolone 2,5g iv in three doses every two days) followed by oral treatment (initial dose of prednisolone $60 \mathrm{mg}$, followed by reduction during next 3 months) was introduced. The therapy led to evident significant regression of symptoms, including regression of eyeballs' protrusion. However, normalization of serum total IgG and IgG4 level was not achieved. Clinical remission remained during 9 months after steroids treatment.

The above therapy (methylprednizolone $3 g$ i.v.) was repeated one year later because of recurrence of symptoms.

Yet, at that moment it was followed by methotrexate treatment (20mg/week).

Until now, 7 months after second glucocorticoids pulse, the patient does not present any symptoms of orbitopathy; well tolerated metotrexate therapy is continued.

Tab.1. Laboratory report

\begin{tabular}{ll|l|}
\hline & Results & Normal range \\
\hline TSH & $1,17 \mathrm{mlU} / \mathrm{L}$ & $0,55-4,78$ \\
\hline FT4 & $1,22 \mathrm{ng} / \mathrm{dl}$ & $0,89-1,76$ \\
\hline FT3 & $3,8 \mathrm{pg} / \mathrm{ml}$ & $2,3-4,2$ \\
\hline Anti-TPO antibodies & $29 \mathrm{Ul} / \mathrm{ml}$ & $0-60$ \\
\hline Thyroglobulin antibodies & $<10 \mathrm{IU} / \mathrm{ml}$ & $<115$ \\
\hline TSH receptor antibodies & $<0,3 \mathrm{IU} / \mathrm{L}$ & $<1,22$ \\
\hline IgG (before steroid treatment) & $2754 \mathrm{mg} / \mathrm{dl}$ & $650-1600$ \\
\hline IgG4(before steroid treatment) & $14 \mathrm{~g} / \mathrm{l}$ & $0,05-1,25$ \\
\hline IgG (after steroid treatment) & $2259 \mathrm{mg} / \mathrm{dl}$ & $650-1600$ \\
\hline IgG4(after steroid treatment) & $13 \mathrm{~g} / \mathrm{l}$ & $0,05-1,25$
\end{tabular}

Fig.1.MRI scans with periorbital muscels involvement

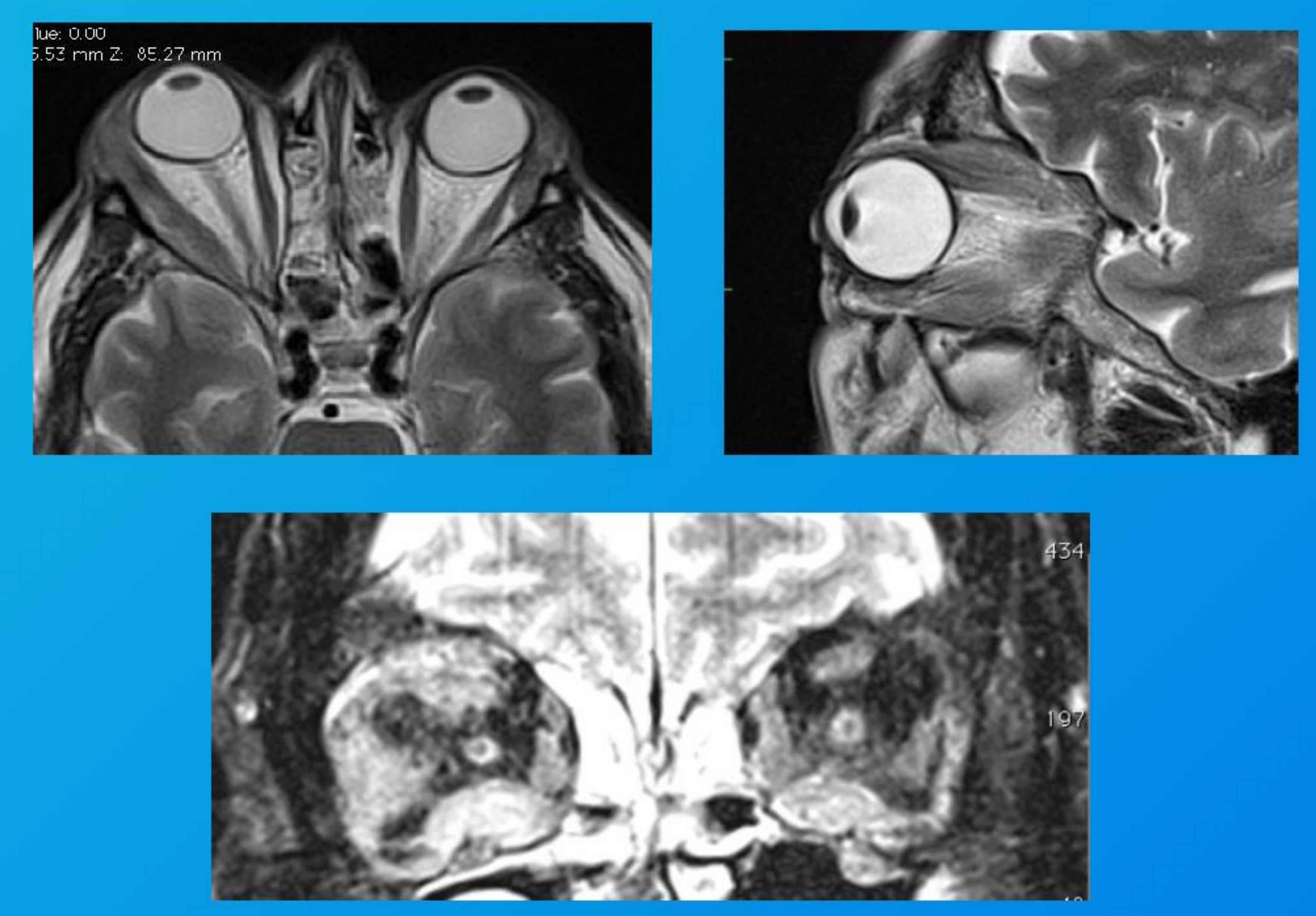

Fig.2.Clinical manifestation of IgG4-related disease

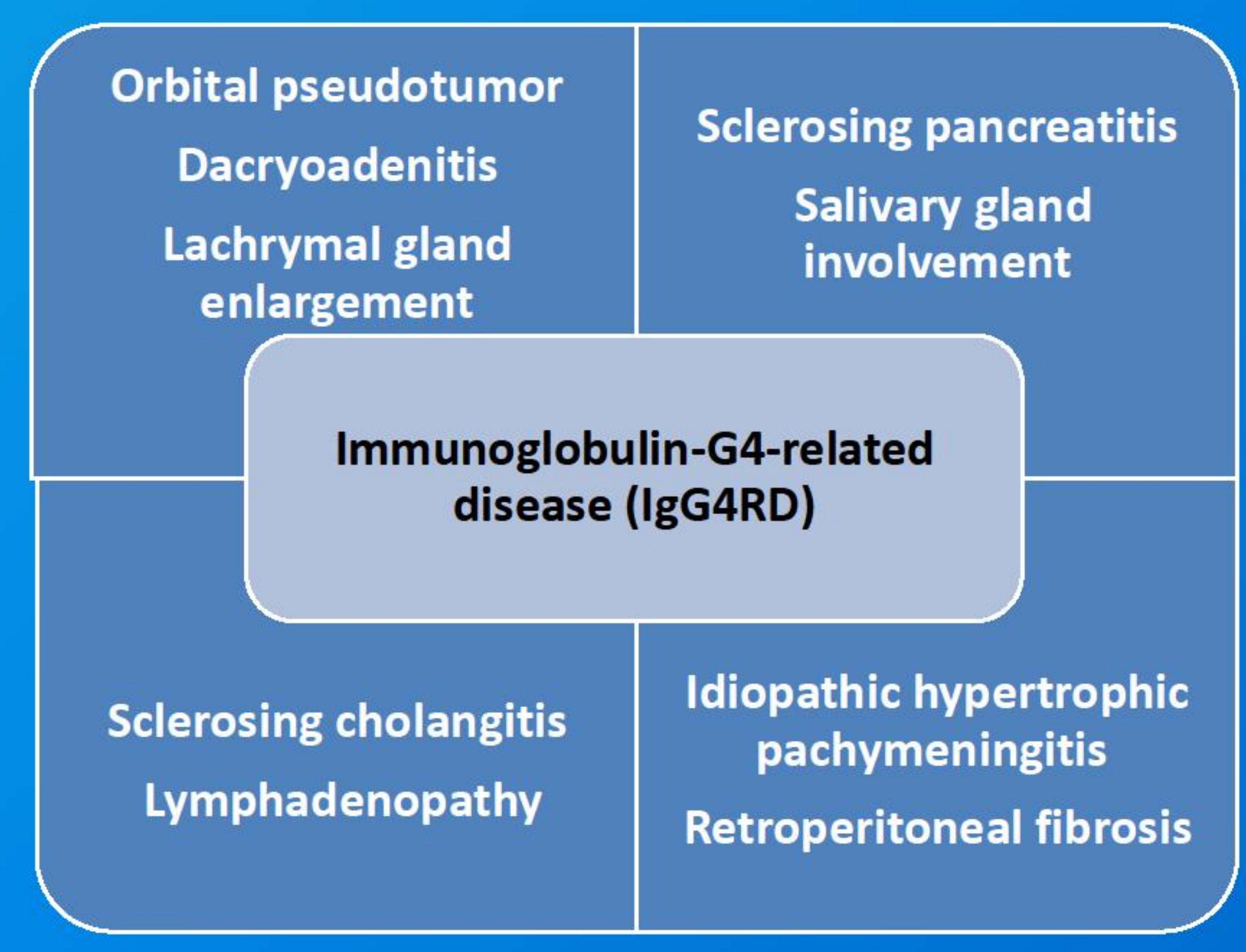

Conclusions:Presented case highlights the importance of considering IgG4RD in the differential diagnosis of orbitopathy.

The 18th European Congress of Endocrinology, 28-31 MAY 2016, Munich, Germany 